\title{
Glucokinase activators (GKAs) promise a new pharmacotherapy for diabetics
}

\author{
Franz M Matschinsky ${ }^{1 *}$ and Daniel Porte $\mathrm{Jr}^{2,3}$
}

\author{
Addresses: ${ }^{1}$ Department of Biochemistry \& Biophysics and Institute for Diabetes, Obesity \& Metabolism, School of Medicine, University of \\ Pennsylvania, 415 Curie Blvd, Clinical Research Bldg, Rm 700, Philadelphia, PA 19104, USA; ${ }^{2}$ Department of Medicine, University of California \\ San Diego, 9500 Gilman Drive, La Jolla, CA 92093-0671, USA; ${ }^{3}$ VA San Diego Healthcare System (111-G), 3350 La Jolla Village Drive, San Diego, \\ CA 92161, USA \\ *Corresponding author: Franz M Matschinsky (matsch@mail.med.upenn.edu) \\ FI000 Medicine Reports 2010, 2:43 (doi:10.3410/M2-43)
}

The electronic version of this article is the complete one and can be found at: http://f $1000 . c o m / r e p o r t s / m e d i c i n e / c o n t e n t / 2 / 43$

\begin{abstract}
The glucose-phosphorylating enzyme glucokinase, a promising target for developing new antidiabetic agents, was identified through the combined efforts of basic research and human biochemical genetics. Allosteric glucokinase activators (GKAs) were discovered by high-throughput screening of a large compound library and first reported in 2003. GKAs stimulate insulin release and glucose metabolism in the liver thereby lowering blood sugar, and promising trials in humans demonstrate that they are highly effective in patients with type 2 diabetes mellitus. Many companies are now attempting to develop effective and safe GKAs for treating diabetics.
\end{abstract}

\section{Introduction and context}

Selecting new targets for developing antidiabetic drugs for type 2 diabetes mellitus (T2DM) is best guided by considerations of established physiological chemistry of glucose homeostasis and of prevailing views about T2DM pathophysiology because the disease genetics that could serve as another guiding principle remain prohibitively perplexing. The glucose-phosphorylating enzyme glucokinase (GK) (also called hexokinase IV or D) was identified as an outstanding drug target for developing antidiabetic medicines because it has an exceptionally high impact on glucose homeostasis, serving as a glucose sensor of the pancreatic beta cell and as a rate-controlling enzyme for hepatic glucose clearance and glycogen synthesis, both processes that are impaired in T2DM [1]. In the mid-1990s, highthroughput screening of 120,000 small molecules of the Roche (Basel, Switzerland) compound library, using a screening assay based on measuring the effect on recombinant GK inhibited by GK regulatory protein (GKRP), identified a lead molecule that stimulated GK directly but also blocked GKRP inhibition of the enzyme [2]. The optimization effort of this newly discovered
GK activator (GKA) molecule resulted in the discovery of more potent, preclinically effective, and apparently safe compounds and culminated in the development of piragliatin (Figure 1), which was advanced to phase II human trials but then shelved for undisclosed reasons [2-6]. The first 2003 report in Science [2] on the development and successful preclinical use of GKAs was soon followed by the issuance of more than 100 patents for GKAs of different chemical structure, demonstrating the keen interest of the pharmaceutical industry in this new class of antidiabetics [7-10]. The report led to the publication of a flood of studies in animal models and the first human trials, contributing significantly to the proof of principle for GKA use as a novel treatment for this disease $[1,3,7-10]$.

\section{Recent advances}

The role of GK in glucose homeostasis

To comprehend the mechanism of action of GKAs and the rationale of using them for treating diabetics, the role of GK at the molecular, cellular, organ, and whole-body levels has to be understood $[1,11,12]$. GK is unique among hexokinase isoenzymes. It is a monomer with a 
Figure I. Chemical structures of glucokinase activators

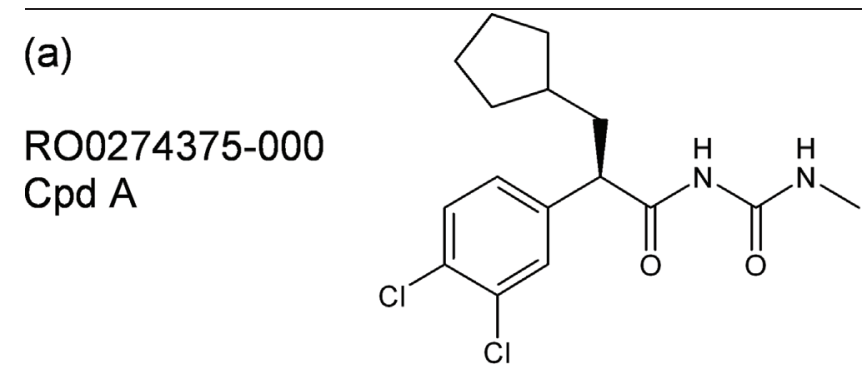

(b)<smiles>[R]O[R]([R])([H])[Se]O[Se]O[O-]</smiles>

(c)
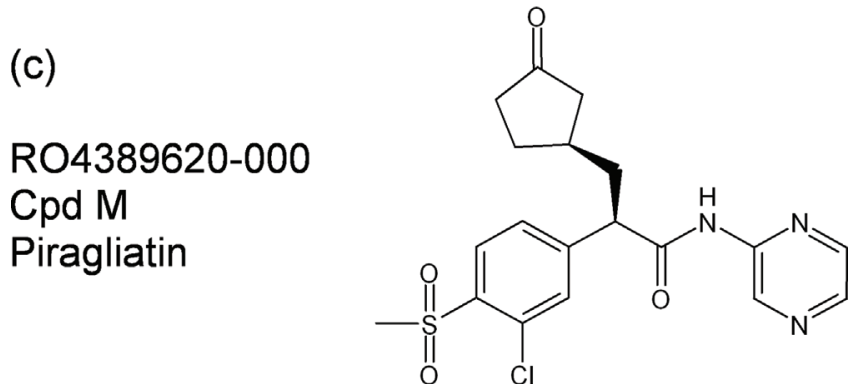

Chemical structures of glucokinase activators used in extensive preclinical studies using (a) RO-0274375-000 (Cpd A) or (b) RO-0281675-000 (Cpd R) and short-term phase II trials in type 2 diabetes mellitus with (c) RO-4389620-000 (piragliatin) [1-6,8-10]. Cpd, compound.

molecular weight of about $50 \mathrm{kDa}$. Its glucose $\mathrm{S} 0.5$ (i.e., the half-saturation concentration) is about $7.0 \mathrm{mM}$, and the co-substrate MgATP saturates physiologically $\left(\mathrm{K}_{\mathrm{m}}=0.4 \mathrm{mM}\right)$. The enzyme shows sigmoidal glucose dependency (has a Hill number of 1.7) and thus is most sensitive to glucose change in the $4.0-\mathrm{mM}$ range. The crystal structure of GK has been solved [13], both as a ligand-free apoenzyme in a 'super-open' conformation and as a ternary complex with one D-glucose and a
GKA bound in a 'closed' conformation (Figure 2). An intermediate so-called 'open' conformation has been postulated to exist. There is strong evidence that the transition between super-open and closed forms is slow compared with the rate of the catalytic cycle, and this evidence is used to explain the physiologically important cooperativity with regard to glucose. Cellular expression of GK is governed by a single gene with two promoters, one of these operating constitutively in GK-containing glucose-sensing endocrine cells (most importantly, in the pancreatic beta cell) and in neurons, and the other controlling hepatic GK expression tightly regulated by insulin. GK thus governs the beta-cell threshold for glucose-stimulated insulin release (GSIR). It accomplishes this by operating in tandem with the adenine nucleotide-sensitive K-channel/SUR1 (sulfonylurea receptor-1) complex and L-type Ca-channels. Alterations of any one of the three beta-cell components influence the threshold for GSIR profoundly. The rate of glucose metabolism as determined by blood glucose levels and the beta-cell GK content is coupled to the two channels: directly by the ATP/ADP ratio to the K-channels and indirectly by the membrane potential to the Ca-channels. High glucose increases GK expression in beta-cells as much as 5- to 10-fold, in a concentration-dependent manner, and thus sensitizes them to glucose stimulation of insulin biosynthesis and release. In excess of $99 \%$ of the bodies' GK protein resides in the liver, explaining its critical role postprandially in the high-capacity clearance of glucose from the blood stream coupled with enhanced glycogen synthesis and glucose catabolism. In hepatocytes, GK is subtly regulated by GKRP, a $68-\mathrm{kDa}$

Figure 2. Effect of glucose and glucokinase activator (GKA) binding on the configuration of the allosteric activator site of glucokinase (GK)

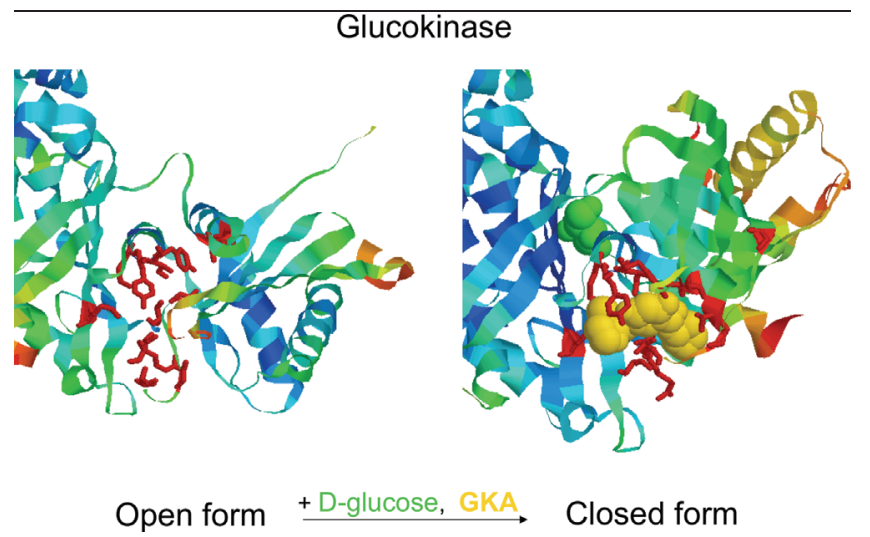

A critical cutout of the GK structure is presented to show the conformational change induced by glucose allowing GKA to bind. Note the marked rearrangement of known contact amino acids in the drug receptor region of the enzyme. Based on structures reported by Kamata et al. [13]. 
primarily nuclear protein that inactivates and facilitates the sequestration of GK in the nucleus when glucose levels are low [14]. High glucose and fructose-1-P (a metabolite of dietary fructose) dissociate the GK/ GKRP complex, which results in reactivation and relocation of GK to the cytosol. Hepatic GK regulates glycogen metabolism, glycolysis, the pentose-P shunt, glucose oxidation, and associated oxidative phosphorylation and ATP production. GK influences hepatic lipid metabolism and gluconeogenesis. The predominant role of this core control system based on beta-cell and hepatic GK is probably complemented in ways to be explored by the function of other GK-containing cells in the hypothalamus, the brain stem, the portal vein, entero- endocrine $\mathrm{K}$ and $\mathrm{L}$ cells, pancreatic islet alpha cells as well as gonadotropes and thyrotropes $[1,11]$ (Figure 3).

\section{GK status in T2DM}

A drug receptor is therapeutically useful only if it is not significantly impaired or deleted by the disease to be treated $[1,15,16]$. What is the status of GK in T2DM? The evidence is strong that beta-cell GK is functional in T2DM, although total GK is probably diminished because beta-cell mass and function are reduced as the disease progresses $[1,16]$. The known right shift and the blunting of the dose-dependency curve of GSIR in T2DM are probably the result of multiple yet-to-be-defined defects of the signaling chains controlling GSIR and

Figure 3. The network of glucokinase (GK)-containing tissues throughout the body and their connections

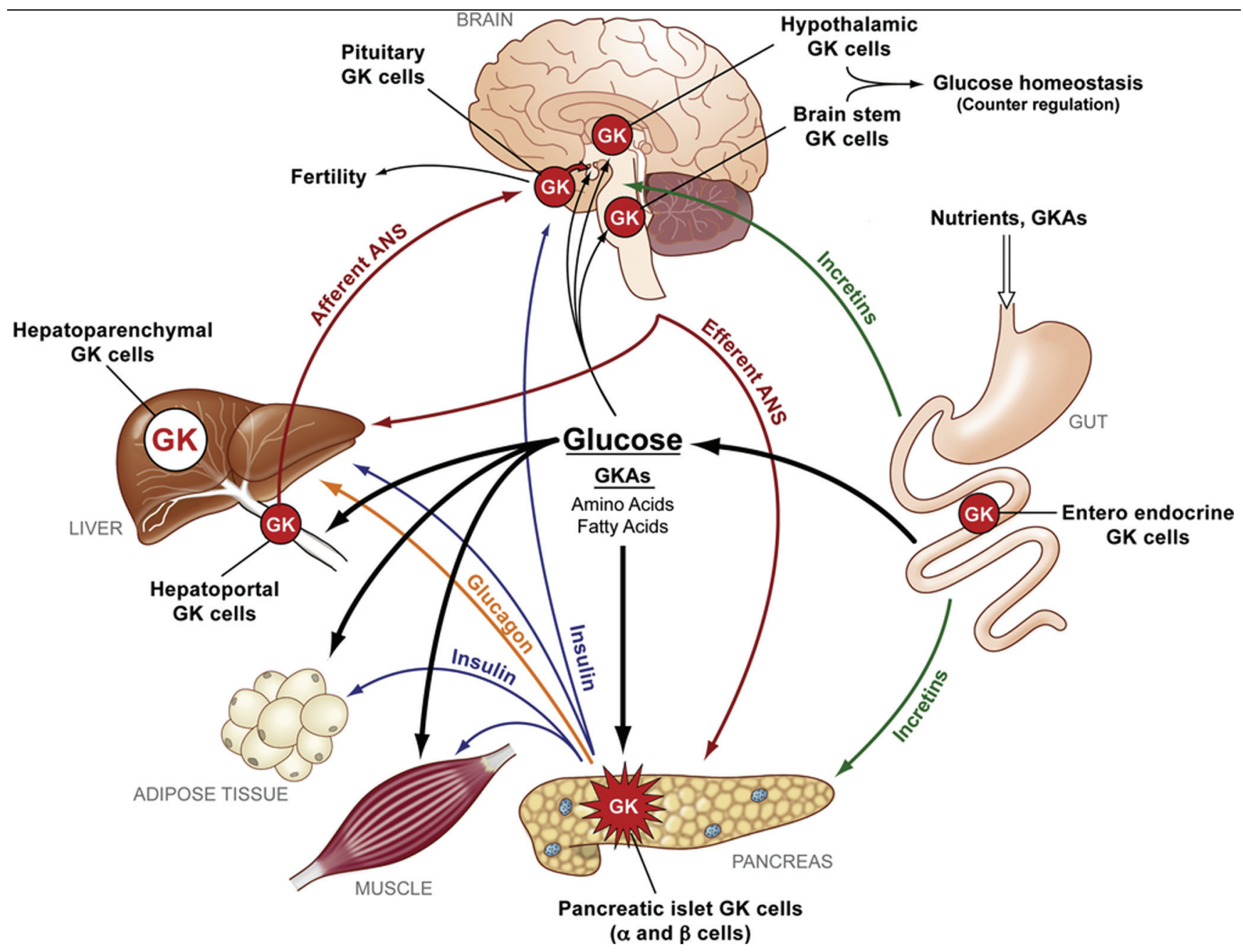

Expression of GK in the pancreatic islet alpha and beta cells, other endocrine cells (including pituitary cells), and cells in the portal vascular tree and central nervous system (including the hypothalamus and brain stem) makes up about $0.1 \%$ of the body's total GK complement. The liver produces the rest (about 99.9\%). The pancreatic islet cells and the liver constitute the basic GK-dependent regulatory system maintaining glucose homeostasis. ANS, autonomic nervous system; GKA, glucokinase activator. Modified from Matschinsky [I], Nat Rev Drug Discov 2009. 
insulin biosynthesis, including perhaps the cellular content or activity of GK (or both). The situation of hepatic GK is quite another matter $[1,15]$. Hepatic GK expression is insulin-controlled and may be greatly reduced in severe forms of T2DM as demonstrated in human and animal models of the disease with severe insulin deficiency. Still, whatever GK remains available or is newly induced by GKA-stimulated insulin release is probably responsive to activation by GKAs. Nothing is known about the status of GK in other cells expressing the enzyme but it is speculated that constitutive expression of GK might be unaltered by the disease.

\section{GKA action at the molecular, cellular, organ, and whole-body levels}

On theoretical grounds, pharmacological activation of GK is expected to have a high potential for the treatment of T2DM and perhaps also T1DM. Early results with GKAs support this view. GKAs are, in general, small molecules with a considerable variety of chemical structures (selected examples in Figure 1) that mostly adhere to a common pharmacophore model with related structural motifs $[1,3,7-10,12]$. The model consists of a center of a carbon or an aromatic ring with three attachments to it. Two of these are hydrophobic, and at least one of the two is aromatic. The third attachment is a 2 -aminoheterocycle or $\mathrm{N}$-acyl urea moiety that provides the basis of forming an electron donor/acceptor interaction with R63 of GK. At least a dozen other amino acids have been shown to be involved in GKA binding: V62, R63, S64, T65, G68, S69, G72, V91, W99, M210, I211, Y214, Y215, M235, V452, V455, and K459. It is noteworthy that mutations of many of these lead to activation of GK and cause hyperinsulinemic hypoglycemia. GKAs increase the affinity of recombinant GK for glucose by as much as 10-fold and may also augment the $\mathrm{V}_{\max }$ (maximum velocity) moderately, perhaps as much as twofold. The affinity for MgATP is increased when tested at glucose levels below the glucose S0.5. Some GKAs lower the Hill coefficient such that they render the enzyme more hexokinase-like. The potency of known GKAs varies considerably, with dissociation constant values ranging from low nanomolars to low micromolars. Most GKAs do not bind to GK in the absence of glucose. GKAs potentiate the competitive glucose reversal of GK inhibition by GKRP. Binding of GKRP and GKAs to GK is mutually exclusive. Little is known about GKA effects on the known interaction of GK with cellular organelles (e.g., mitochondria or insulin granules) or other macromolecules besides GKRP (e.g., the proapoptotic BAD [Bcl-2-associated death promoter] protein $[17,18]$ or the bifunctional enzyme that generates and hydrolyzes fructose-2,6-bisphospate [19]). Impaired formation of a GK/BAD complex proposed to associate with mitochondria and to enhance glucose oxidation may be significant for the molecular pathogenesis of the functional beta-cell defect and apoptotic beta-cell loss known to cause T2DM. It is tempting to hypothesize that GKAs might remedy this defect. Indeed, as reported at the 2009 International Diabetes Federation meeting in Montreal (Matschinsky et al., unpublished data), studies from our laboratory have demonstrated a marked reduction of glucose-induced respiration in islets isolated from T2DM organ donors and showed that this metabolic defect was partially or fully remedied in acute studies using the Hoffmann-La Roche GKA piragliatin. Therefore, it is not unreasonable to explore whether GKAs are anti-apoptotic in GK-containing cells. As we showed in these studies with islets of Langerhans isolated from the pancreas of rodents or of normal and diabetic human tissue donors, GKAs potentiate glucose stimulation of insulin release and enhance the permissive action of glucose for other physiological stimuli, including amino acids, fatty acids, acetylcholine, and GLP-1 (glucagon-like peptide-1). GKAs markedly potentiate glucose induction of islet GK expression. It can be extrapolated that they increase glucose stimulation of insulin biosynthesis. Studies with isolated rat hepatocytes demonstrated that GKAs stimulate glycolysis and glycogen synthesis and effectively dissociate the nuclear GK/GKRP complex, thus activating glucose phosphorylation $[3,15,20]$.

In intact control animals and a wide variety of animal models of T2DM, GKAs uniformly lowered blood glucose and stimulated insulin release [1-3,7-10]. They also curbed hepatic glucose production in normal and diabetic rats. In a long-term study with C57black6J mice, GKAs prevented the development of diet-induced diabetes with little effect on the weight gain of the animals [21]. Results of human trials with normal or diabetic patients showed that GKAs reduced blood glucose dose-dependently and increased insulin secretion. The blood glucose-lowering potential of GKAs in T2DM is compellingly illustrated by results of trials with piragliatin from Roche in a 5.5-day multiple ascending dose study $[5,6,17]$ and a 1-day single ascending dose study with ARRY403 from Array BioPharma (Boulder, CO, USA) [22]. Moderate hypoglycemia was observed at the highest dosages.

\section{Implications for clinical practice}

The published results of treating T2DM patients with GKAs for periods of up to 1 week demonstrate that these agents lower blood glucose effectively in a dosedependent manner without medically significant side actions except (not surprisingly) moderate hypoglycemia at the higher doses. Thus, intensive research and development have produced a class of powerful 
antidiabetic drugs with a new mechanism of action, contrasting it to all other available pharmacotherapies, including insulin. It remains to be seen whether this novel class of drug withstands the test of time and secures for itself a significant place among approved antidiabetic medicines. The massive current effort of at least 15 pharmaceutical companies to develop GKAs shows that expectations for success in this endeavor are high. Of remaining concern are the dangers of hypoglycemia, fatty liver, and hyperlipidemia. Available results of preclinical and clinical studies are, however, reassuring in this regard and it is reasonable to argue that such potential side effects can be managed by designing GKAs, which are less potent than the currently known prototypes, or by judicious dose regimens. All indications are that several GKAs now in development will be advanced to phase II and III trials [1-10]. Beyond their potential medical significance, GKAs will also prove highly useful as an experimental tool in studies of the GK molecule and of glucose homeostasis in health and disease (Figure 3).

\section{Abbreviations}

$\mathrm{BAD}, \mathrm{Bcl}-2$-associated death promoter; $\mathrm{GK}$, glucokinase; GKA, glucokinase activator; GKRP, glucokinase regulatory protein; GSIR, glucose-stimulated insulin release; T2DM, type 2 diabetes mellitus.

\section{Competing interests}

The authors declare that they have no competing interests.

\section{Acknowledgments}

These studies were supported by National Institutes of Health grants: National Institute of Diabetes and Digestive and Kidney Diseases (NIDDK) 22122 and 19525.

\section{References}

I. Matschinsky FM: Assessing the potential of glucokinase activators in diabetes therapy. Nat Rev Drug Discov 2009, 8:399-4I6.

2. Grimsby J, Sarabu R, Corbett WL, Haynes N-E, Bizzarro FT, Coffey JW, Guertin KR, Hilliard DW, Kester RF, Mahaney PE, Marcus L, Qi L, Spence CL, Tengi J, Magnuson MA, Chu CA, Dvorozniak MT, Matschinsky FM, Grippo JF: Allosteric activators of glucokinase: potential role in diabetes therapy. Science 2003, 301:370-3.

FI000 Factor 3.0 Recommended

Evaluated by Barry Stoddard 20 Aug 2003

3. Pal M: Recent advances in glucokinase activators for treatment of type 2 diabetes. Drug Discovery Today 2009, I 4:784-92.

4. Zhi J, Zhai S, Mulligan ME, Grimsby J, Arbet-Engels C, Boldrin M, Balena R: A novel glucokinase activator RO4389620 improved fasting and postprandial plasma glucose in type 2 diabetic patients. Diabetologia 2008, 5 I (SuppI I):S23. Abstract 42.

5. Bonadonna RC, Kapitza C, Heinse T, Avogaro A, Boldrin M, Grimsby J, Mulligan ME, Arbet-Engles C, Balena R: Glucokinase activator RO4389620 improves beta cell function and plasma glucose indexes in patients with type 2 diabetes. Diabetologia 2008, 5 I (Suppl I):S37I. Abstract 927.

6. Zhai S, Mulligan ME, Grimsby J, Arbet-Engels C, Boldrin M, Balena R, Zhi J: Phase I assessment of a novel glucose activator RO4389620 in healthy male volunteers. Diabetologia 2008, 5 I (Suppl I):S372. Abstract 928.

7. Coghlan $M$, Leighton $B$ : Glucokinase activators in diabetes management. Expert Opin Investig Drugs 2008, I 7:145-67.

8. Guertin KR, Grimsby J: Small molecule glucokinase activators as glucose lowering agents: a new paradigm for diabetes therapy. Curr Med Chem 2006, I3:1839-43.

9. Sarabu R, Berthel SJ, Kester RF, Tilley JW: Glucokinase activators as new type 2 diabetes therapeutic agents. Expert Opin Ther Pat 2008, 18:759-68.

10. Sarabu R, Grimsby J: Targeting glucokinase activation for the treatment of type 2 diabetes - a status review. Curr Opin Drug Discov Devel 2005, 8:63I-7.

II. Matschinsky FM, Magnuson MA, Zelent D, Jetton TL, Doliba N, Han Y, Taub R, Grimsby J: The network of glucokinase-expressing cells in glucose homeostasis and the potential of glucokinase activators for diabetes therapy. Diabetes 2006, 55: I-I 2 .

12. Matschinsky FM: Banting Lecture 1995. A lesson in metabolic regulation inspired by the glucokinase glucose sensor paradigm. Diabetes 1996, 45:223-4I.

13. Kamata K, Mitsuya M, Nishimura T, Eiki J, Nagata Y: Structural basis for allosteric regulation of the monomeric allosteric enzyme human glucokinase. Structure 2004, I 2:429-38.

14. Veiga-da-Cunha M, Van Schaftingen E: Identification of fructose 6-phosphate- and fructose I-phosphate-binding residues in the regulatory protein of glucokinase. J Biol Chem 2002, 277:8466-73.

15. Agius L: Glucokinase and molecular aspects of liver glycogen metabolism. Biochem J 2008, 4|4: I-I8.

16. Del Guerra S, Lupi R, Marselli L, Masini M, Bugliani M, Sbrana S, Torri S, Polera M, Boggi U: Functional and molecular defects of pancreatic islets in type 2 diabetes. Diabetes 2005, 54:727-35.

17. Danial NN, Gramm CF, Scorrano L, Zhang C-Y, Krauss S, Ranger AM, Datta SR, Greenberg ME, Licklider LJ, Lowell BB, Gygi SP, Korsmeyer SJ: BAD and glucokinase reside in a mitochondrial complex that integrates glycolysis and apoptosis. Nature 2003, 424:952-6.

FI000 Factor 4.8 Must Read

Evaluated by Atan Gross 29 Aug 2003, Kermit Carraway 09 Sep 2003

18. Danial NN, Walensky LD, Zhang C-Y, Choi CS, Fisher JK, Molina AJA, Datta SR, Pitter KL, Bird GH, Wikstrom JD, Deeney JT, Robertson K, Morash J, Kulkarni A, Neschen S, Kim S, Greenberg ME, Corkey BE, Shirihai OS, Shulman GI, Lowell BB, Korsmeyer SJ: Dual role of proapoptotic BAD in insulin secretion and beta cell survival. Nature Med 2008, I 4:144-53.

19. Baltrusch S, Wu C, Okar DA, Tiedge M, Lange AJ: Interaction of GK with the bifunctional enzyme 6-phosphofructo-2-kinase/ fructose-2,6-bisphosphatase (6PF2K/F26P2ase). In Glucokinase and Glycemic Disease: From Basics to Novel Therapeutics. Front Diabetes Vol. I6. Edited by Matschinsky FM, Magnuson MA. Basel: Karger; 2004 : 262-74.

20. Brocklehurst KJ, Payne VA, Davies RA, Carroll D, Vertigan HL, Wightman HJ, Aiston S, Waddell ID, Leighton B, Coghlan MP, Agius L: Stimulation of hepatocyte glucose metabolism by novel small molecule glucokinase activators. Diabetes 2004, 53:535-4I.

21. Grimsby J, Matschinsky FM, Grippo JF: Discovery and actions of glucokinase activators. In Glucokinase and Glycemic Disease: From Basics to Novel Therapeutics. Front Diabetes. Vol. 16. Edited by Matschinsky FM, Magnuson MA. Basel: Karger; 2004:360-78.

22. Array BioPhama: Glucokinase Activator ARRY-403: Phase I Single Ascending Dose Top-Line Results. August 10, 2009. [http://www.arraybiopharma.com/Documents/PDF/Slides.pdf] 